J. Environ. Sci.

Institute of Environmental Studies and Research - Ain Shams University

\title{
DETECTION OF MELANOMA USING IMAGE PROCESSING TECHNIQUES DRIVEN TDS INDEX
}

Mohamed S. Hassan ${ }^{(1)}$; Noha S. Donia ${ }^{(1)}$; Tarek A. Mahmoud ${ }^{(2)}$ and May H. Elsamahy ${ }^{(3)}$

1) Institute of Environmental Studies and Research, Ain Shams University 2) Military Technical College (MTC), Cairo, Egypt 3) Dermatology, Venereology and Andrology Department, Medicine College, Ain Shams University

\begin{abstract}
Skin cancer exists in different forms such as: Melanoma, Basal and Squamous Cell Carcinoma, among which Melanoma is the most dangerous and unpredictable skin cancer. In this paper, we implement an image processing technique for the detection of Melanoma skin cancer, in which the input to the system is the skin lesion image. This image proceeds with the image preprocessing methods such as conversion of (Red, Green, Blue)RGB image to gray scale image, noise removal, hair removal and so on. Moreover thresholding is used to segment the images followed by feature extraction that includes parameters such as: Asymmetry, Border irregularity, Color and Diameter (ABCD). Finally, the total Dermoscopic score (TDS) is calculated and decision is to be taken whether the cancer is Melanoma or not.
\end{abstract}

Keywords: Skin lesion, Melanoma, TDS, ABCD Parameters

\section{MEDICAL INTRODUCTION}

Image processing is the processing of images using any form of signal processing for which the input is an image, or a series of images, or a video. Skin cancers are cancers that arise from the skin. They are due to the development of abnormal cells that have ability to invade or spread to other parts of the body. Skin cancer is found in various types such as Basal cell skin cancer (BCC), Squamous cell carcinoma (SCC) and Melanoma among which 
Melanoma is the most unpredictable skin cancer. Melanoma begins in melanocytes (Pigment Cells). Most melanocytes are in the skin and thus Melanoma can occur on any skin surface (Ramya et al., 2015).

In men, it is often found on the skin on the head, on the neck or between the shoulders and the hips. In women, it is often found on the skin on the lower legs or between the shoulders and the hips. Melanoma is rare in people with dark skin. When it does develop in people with dark skin, it is usually found under the finger nails, under the toe nails. On the palms of the hands, or on the soles of the feet (Ramya et al., 2015).

If Melanoma is recognized in the early stages it is proven to be curable, if not, the cancer advances and spreads to all other parts of the body and becomes incurable leading to death. It is estimated 76,690 people being diagnosed with Melanoma and 9,480 people died by Melanoma in the United States in 2013. Melanoma is more likely than other skin cancers to spread to other parts of the body (Ramya et al., 2015). Therefore in this work, Melanoma skin cancer is implemented using image processing such as segmentation and feature extraction techniques. 
J. Environ. Sci.

Institute of Environmental Studies and Research - Ain Shams University

\section{Detection of Melanoma Skin Cancer Using TDS Calculation Method:}

Fig. 1 introduces the steps of the TDS calculation method for the detection of Melanoma skin cancer.

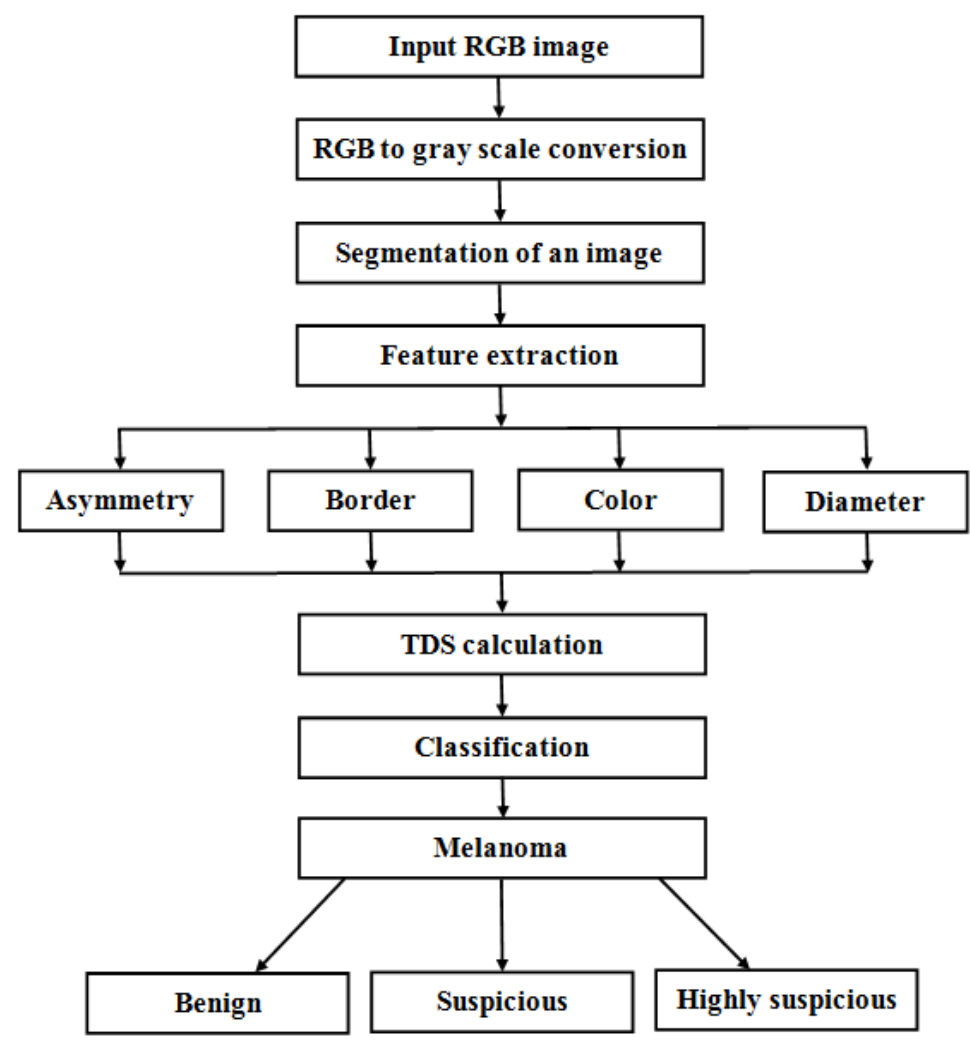

Fig.1: Block diagram of the proposed method by using segmentation and feature extraction techniques

\section{Methodology:}

(1) Input image: Image acquisition in image processing can be broadly defined as the action of retrieving an image from some source, usually hardware based source. So that it can be passed through whatever processes 
need to occur afterward. Performing image acquisition in image processing is always the first step in the workflow sequence because, without an image, no processing is possible. The input to the system is the skin lesion image which is taken from the medical database.

(2)- RGB to gray scale conversion: Image enhancement includes image scaling color normalization and contrast enhancement. Generally, the common color spaces are RGB, HSV, CIE LAB and CIE XYZ. In this technique, the ordinary image is first converted to RGB and then to gray scale. It makes an image suitable for a particular application such as in our case.

Furthermore, contrast enhancement takes place. Contrast enhancement is a beneficial step to improve the perception for further processing such as sharpening the image border, improving image brightness. At the end of the preprocessing step of skin cancer detection system, the resulting images are distinguishable from those initial images and almost are ready to feed the segmentation stage (Aswini et al.,2013). 
(3)-Segmentation of an image: The process of segmentation is to make things easier or change the representation of an image into something that is more understandable and simpler to analyze. Segmentation refers to the separation of an image into disjoint regions that are uniform with respect to some property such as color luminance and texture (Jamil et al., 2015) . The segmentation techniques used for a dermoscopic images are thresholding, color based segmentation, clustering techniques, histogram equalization, discontinuity segmentation, Otsu method and so on. In this paper, the segmentation technique used is Otsu thresholding based segmentation. Otsu thresholding is a clustering based image segmentation technique or reduction of a gray scale image to a binary image which will be explained later.

(4)-Feature extraction: In order to educate the masses to recognize Melanoma in its early stage in 1985, group from New York University devised the ABCD acronym (Asymmetric, Border irregularity, Color, Diameter) (Ahmed et al., 2014).

(a) Asymmetry: cancerous lesions are checked for symmetry in 0, 1or 2 axes. It is considered as 0-biaxial symmetry, 1-mono axial symmetry, 2-biaxial asymmetry.

(b)Border irregularity: most of the cancerous lesions are ragger, notched or blurred. The border irregularity value ranges from 0 to 8 . Border irregularity can be calculated using the following formula in equation (1):

$$
B=[(\text { perimeter }) .(\text { perimeter }) / 4 * 3.14 * A] \ldots \ldots . .(1)
$$

Where, $\mathrm{A}=$ Area of the lesion. (Using image region analyzer) 
(c)-Color: cancerous skin lesions pigmentation is not uniform. The presence of up to 6 known colors must be detected- White, Red, Light brown, Dark brown, Blue gray and Black. The color value ranges from 0 to 6 .

(d)-Diameter: cancerous lesions are greater than $6 \mathrm{~mm}$ wide. Differential structures with at least five patterns are relevant for specific types of lesions. Any growth of a mole should be of concern. The diameter value ranges from 0 to 5 ((Ramteke et al., 2013).

$\mathrm{ABCD}$ rule has proven more accurate and effectiveness in clinical practice with high diagnostic accuracy. The $\mathrm{ABCD}$ rule is also used by the American Cancer Society, American Academy of Dermatology and others worldwide to provide simple parameters for evaluation and identification of pigmented lesions that may need further examination (Bhardwaj et al., 2014). Otsu and Stolz algorithms (Iqbal et al., 2015) are explained in the next section.

\section{Algorithm Description:}

(a)Otsu's algorithm: This stage introduces segmentation step by performing Otsu segmentation on the gray scale image.

Step (1): Prepare the input RGB image.

Step (2): Convert RGB image to gray scale image.

Step (3): Add noise to the image.

Step (4): Median Filter $(3 * 3)$ is used to remove the noise from the image.

Step (5): Use Otsu segmentation method to segment the image.

(b)Stolz's algorithm: This stage prepares the feature extraction parameters by Stolz's algorithm, which is then used to calculate the TDS value in the last stage. 
Step (1): Convert the segmented image into binary image.

Step (2): Calculate Asymmetry of the binary image

Step (3): Calculate the border irregularity of the binary image by calculating the area and perimeter of the lesion.

Step (4): Calculate the color variation and diameter of the lesion.

Step (5): Find the total dermatoscopic score (TDS) by substituting all the parameters with the calculated values.

(c)TDS Calculatio: Dermoscopic images are basically digital images of magnified skin lesion, taken with conventional camera equipped with special lens extension. The lens attached to the dermatoscope acts like a microscope magnifier with its own light source that illuminates the skin surface evenly. Digital images acquired using photo dermatoscope are sufficiently high resolution to allow for precise analysis in terms of differential structures appearance.

Dermatologist can create accurate documentation of gathered images, opening a path for computer analysis, where images are processed in order to extract information that can later be used to classify those images using TDS method (Jaiswar et al., 2015).

TDS (Total Dermatoscopy Score) index is an important tool used in the diagnosis of Melanoma. Calculation of the TDS index is based on Asymmetry, Border irregularity, Color and Diameter of the skin lesion. Each of the criteria is then multiplied by a given weight factor to yield a Total Dermatoscore Score. 
The TDS index is computed using the formula in equation (2):

$$
\mathrm{TDS}=1.3 \mathrm{~A}+0.1 \mathrm{~B}+0.5 \mathrm{C}+0.5 \mathrm{D}
$$

If the TDS index is less than 4.75 , it is benign (non-cancerous) skin lesion.

If the TDS index is greater than 4.75 , less than 5.45 , it is suspicious case of skin lesion.

If the TDS index is greater than 5.45, it is malignant Melanoma (cancerous) skin lesion (Jaiswar et al., 2015).

\section{EXPERIMENTAL RESULTS}

The proposed fusion method is applied on four sample images (image1, image2, image3, image4), the samples of the dermoscopy images used can be downloaded at http; //www.dermnet.com. The algorithm concludes about the presence of Melanoma skin cancer by classifying it as benign, suspicious or highly suspicious skin lesion according to the following steps:

Step (1): Input image (RGB image).

Step (2): RGB to gray scale conversion as shown in Fig.2.

Step (3):Segmentation of the image using Otsu segmentation as shown in

Fig.3.

Step (4): Feature extraction using Stolz's algorithm.

Step (5): TDS calculation.

Step (6): Determination of cancerous image as shown in Table 1. 
J. Environ. Sci.

Institute of Environmental Studies and Research - Ain Shams University

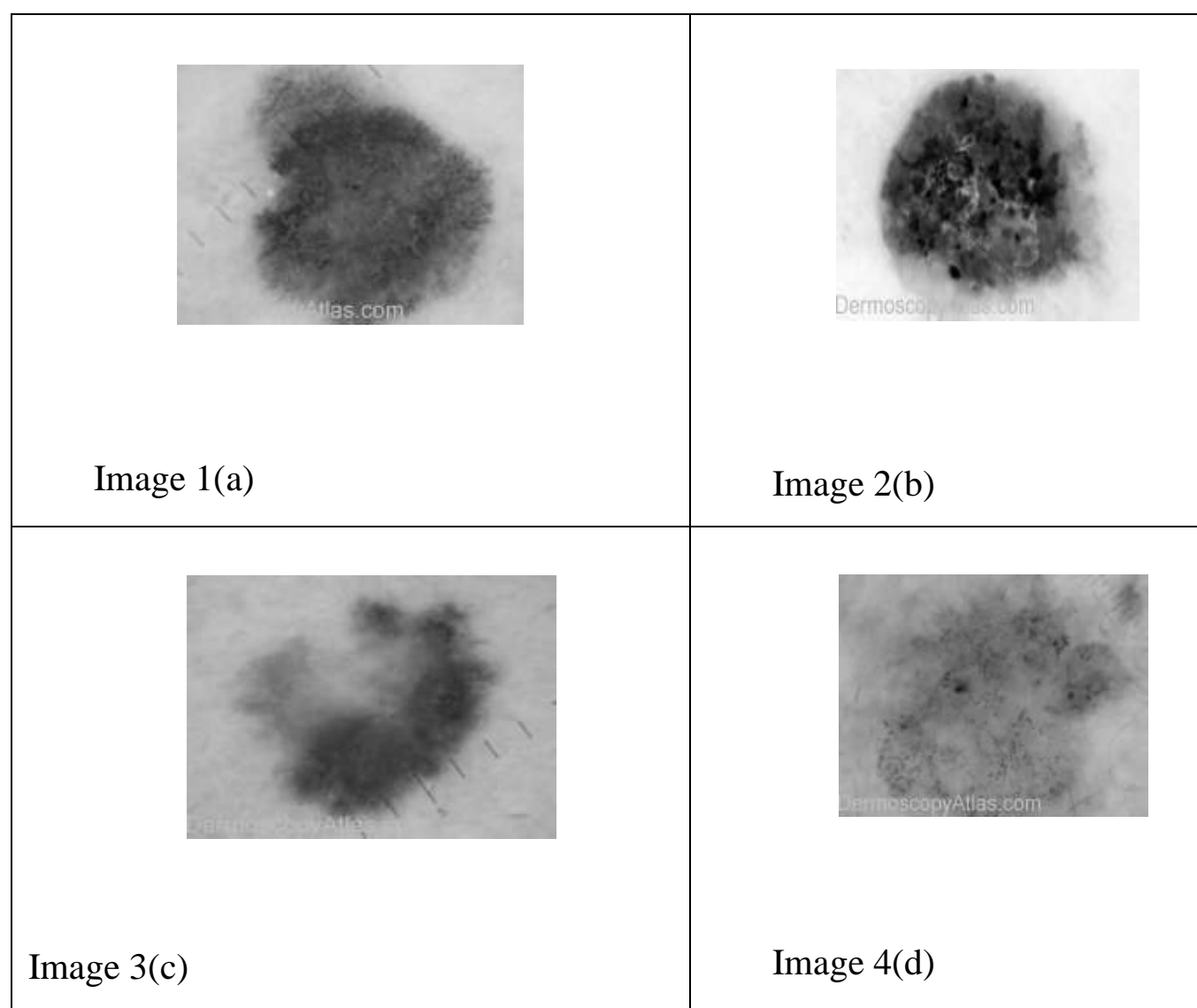

Fig.2: RGB to Gray Scale (Image Conversion)

Table 1: TDS Calculation

\begin{tabular}{|c|c|c|}
\hline Image & TDS & Decision \\
\hline \hline Image 1 & 6.2753 & Highly Suspicious Melanoma \\
\hline Image 2 & 6.2945 & Highly Suspicious Melanoma \\
\hline Image 3 & 4.8970 & Suspicious Melanoma \\
\hline Image 4 & 5.1738 & Suspicious Melanoma \\
\hline
\end{tabular}




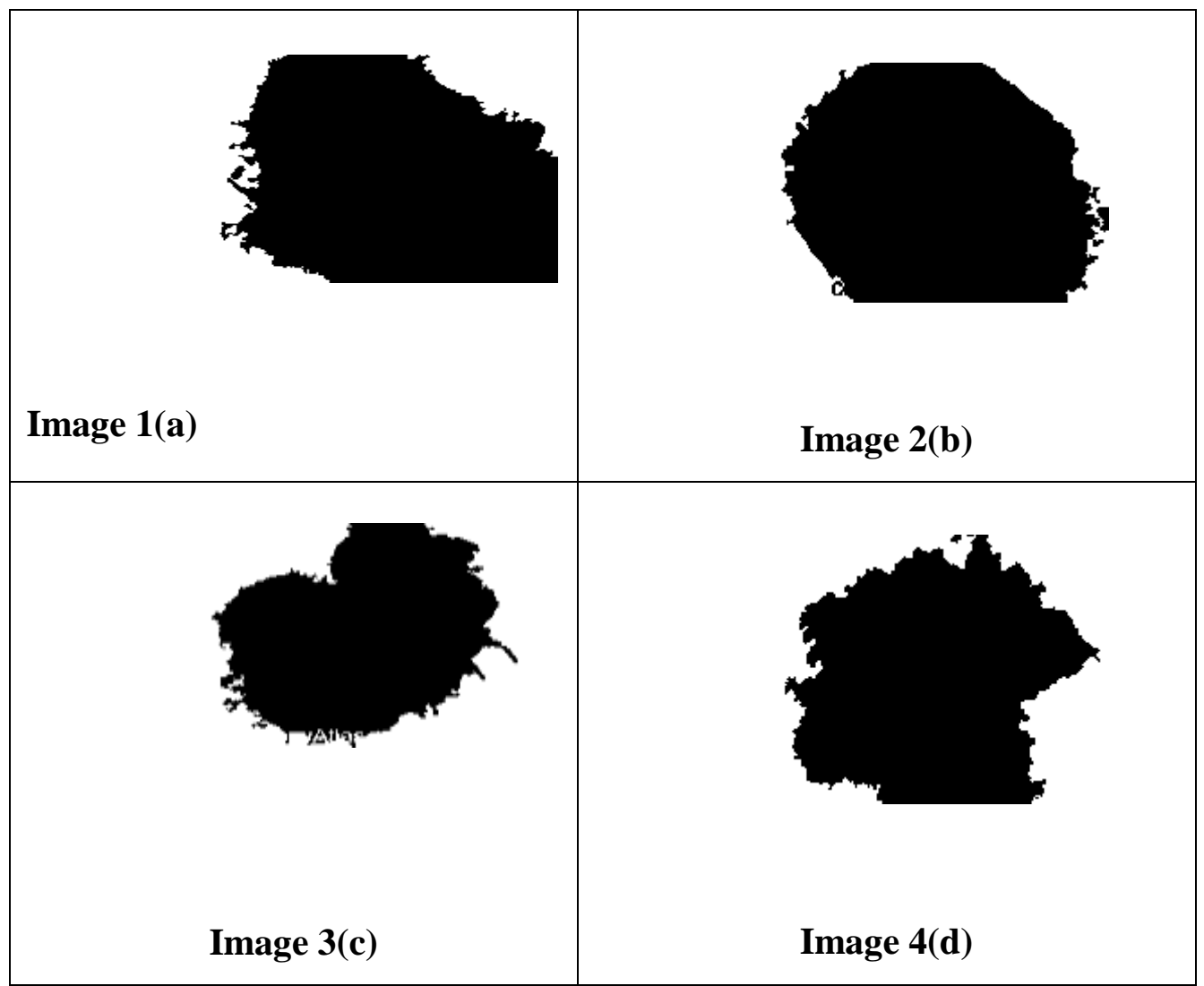

Fig.3: Segmented Images

\section{CONCLUSIONS}

Melanoma is a serious and sometimes life threatening cancer. It can occur in any part of the body that contains melanocytes. The main cause of melanoma is excessive exposure to ultraviolet radiation reaching the skin. Ultraviolet rays from the sun and other sources can damage skin cells, causing the cells to grow abnormally. Early detection of cancer can help the patients to have a proper treatment. The proposed method detects Melanoma skin cancer with proper accuracy. The final output given by the system will 
help the dermatologist to detect the lesion and its type. Accordingly with his knowledge and with the aid of the proposed system, he will examine the patient to draw a final conclusion whether it can be operated or not or any other ways to cure it for example: using medicines or ointments, etc. Skin cancer detection system will help dermatologist to diagnose melanoma in early stages.

\section{REFERENCES}

Ahmed, I.N.; Chaya, p.(2014): Segmentation and Classification of skin cancer images. International Journal of Advanced Research in Computer Science and Software Engineering,4(5),1349-1353.

Aswini, A.; Cirimala, E.; Ezhilarasi, R. and Jayapratha, M.(2013): Noninvasive Screening and Discrimination of Skin Images for Early Melanoma Detection. International Journal of Scientific Research and Management,2(4),781-786.

Bhardwaj, A.; Bhatia, J.S.(2014): An Image Segmentation Method for Early Detection and Analysis of Melanoma. IOSR Journal of Dental and Medical Sciences, 13(10),18-22.

Iqbal, S.; Divyashree, J.A.; Sophia, M.; Mundas, M. and Vidya, R.(2015): Implementation of Stolz's Algorithm for Melanoma Detection. International Advanced Research Journal in Science,Engineering and Technology,2(6),9-12.

Jaiswar, S.; Kadri, M. and Gatty, V.(2015): Skin Cancer Detection using Digital Image Processing. International Journal of Scientific Engineering and Research,3(6),138-140.

Jamil, U. and Khali, S.(2015): Valuable Preprocessing and Segmentation Technique used in Automated Skin Lesion Detection System. $17^{\text {th }}$ UKSIM-AMSS International Conference on Modeling and Simulation,290-295. 
Ramya, V.J.; Navrajan, J; Prathipa, R. and Kumar, L.A.(2015): Detection of Melanoma Skin Cancer using digital camera images. ARPN Journal of Engineering and Applied Sciences, 10(7), 3082-3085.

Ramteke, N.S. and Jain, S.V.(2013): ABCD Rule Based Automatic Computer Aided Skin Cancer Detection using Matlab. International Journal of Computer Technology and Applications,4(4),691-697.

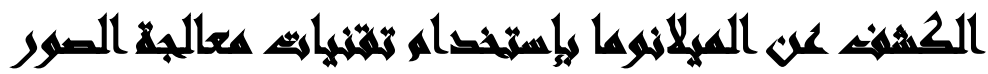

$[Y]$

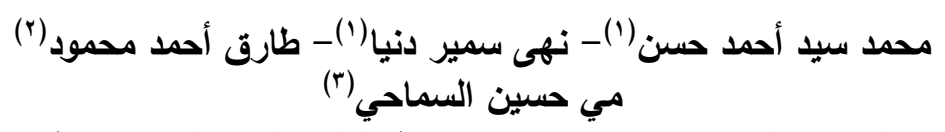

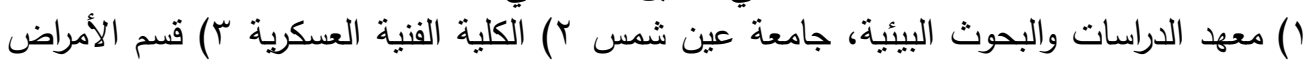

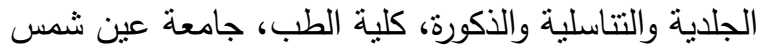

\section{lill}

يظهر سرطان الجلد في صور مختلفة منها الميلانوما وسرطان الخلايا القاعدية وسرطان

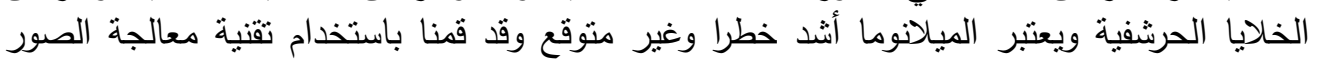

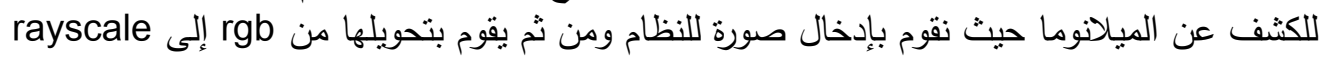

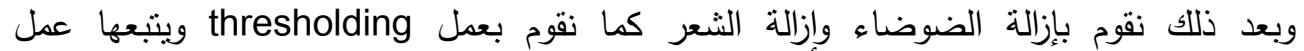
segment

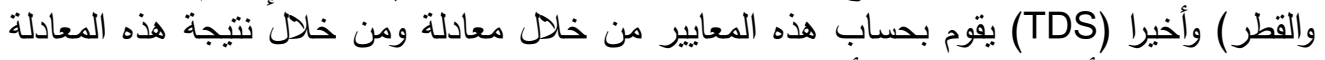

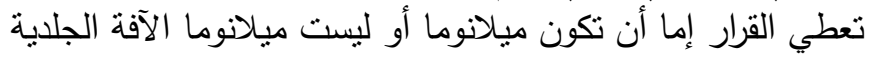
الكلمات الدالة: ABCD معايير \& \& TDS \& ميلانوما 